\title{
Limited Sampling Model for Advanced Mycophenolic Acid Therapeutic Drug Monitoring After Liver Transplantation
}

\author{
Pieter Langers, MD, PhD, * Rogier R. Press, PharmD, PhD, $\dagger$ Akin Inderson, MD,* \\ Serge C. L. M. Cremers, PharmD, PhD, $\ddagger$ Jan den Hartigh, PharmD, PhD, $†$ \\ Andrzej G. Baranski, MD, PhD, $\S$ and Bart van Hoek, MD, PhD*
}

\begin{abstract}
Background: The immunosuppressive drug mycophenolate mofetil (MMF), with mycophenolic acid (MPA) as active metabolite, is a nonnephrotoxic alternative to calcineurin inhibitors. Therapeutic drug monitoring (TDM) of MPA may improve clinical benefit from MMF therapy, especially in MMF monotherapy or with reduced dose of a calcineurin inhibitor. Limited data are available on TDM strategies for MPA in orthotopic liver transplantation (OLT). The authors here describe the pharmacokinetic (PK) behavior of MPA after OLT and developed a Bayesian limited sampling model for monitoring MMF after OLT.
\end{abstract}

Methods: PK data were obtained from 57 stable patients, and trapezoidal area under the curve $\left(\mathrm{AUC}_{0-12 \mathrm{~h}}\right)$ was calculated. The effect of the covariates kidney function and serum albumin concentration was studied. A TDM strategy was developed based on individualized population PKs using Bayesian estimations and limited sampling models to predict the MPA AUC.

Results: A relationship between MMF dose and MPA AUC was found and a 8-fold apparent clearance range of MPA was observed at the same dose level. Significant relationships of albumin concentration and creatinine clearance with MPA plasma clearance were identified (respectively, $\mathrm{r}^{2}=0.12$ and $0.24 ; P<0.05$ ). A model with limited sampling at $0,0.5,1,2$, and 3 hours after drug administration showed very good correlation with trapezoidal $\mathrm{AUC}_{0-12 \mathrm{~h}}$ with acceptable bias and precision $\left(\mathrm{r}^{2}=0.92\right.$, mean prediction error $=1$, mean absolute prediction error $=13 ; P<0.05$ ).

Conclusions: Remarkable variability of MPA clearance in stable OLT patients exists, which can be partially explained by the patients' albumin serum levels and creatinine clearance. Systemic exposure in these patients can be accurately assessed by the Bayesian limited sampling TDM strategy.

Received for publication August 16, 2012; accepted July 3, 2013.

From the Departments of *Gastroenterology and Hepatology; and $\dagger$ Clinical Pharmacy and Toxicology, Leiden University Medical Center, The Netherlands; $\ddagger$ Department of Medicine, Columbia University, New York, NY; and §Department of Transplant Surgery, Leiden University Medical Center, The Netherlands.

P. Langers and R. R. Press have contributed equally.

Supplemental digital content is available for this article. Direct URL citations appear in the printed text and are provided in the HTML and PDF versions of this article on the journal's Web site (www.drug-monitoring.com).

Correspondence: Bart van Hoek, MD, PhD, Department of Gastroenterology and Hepatology, C4-P, Leiden University Medical Center, PO Box 9600, 2300 RC Leiden, The Netherlands (e-mail: b.van_hoek@lumc.nl).

Copyright $(C) 2014$ by Lippincott Williams \& Wilkins
Key Words: orthotopic liver transplantation, therapeutic drug monitoring, mycophenolate mofetil, mycophenolic acid, limited sampling models

(Ther Drug Monit 2014;36:141-147)

\section{INTRODUCTION}

The immunosuppressive agent mycophenolate mofetil (MMF) is the 2-morpholinoethyl ester of mycophenolic acid (MPA), the active metabolite. MPA is an inosine monophosphate dehydrogenase inhibitor and therefore inhibits the de novo pathway of guanosine nucleotide synthesis and thus the proliferative responses of T- and B-lymphocytes. ${ }^{1} \mathrm{MMF}$ is widely used as immunosuppressive drug after different types of organ transplantation, including orthotopic liver transplantation (OLT). It is often administered in combination with dose reduction or cessation of a calcineurin inhibitor (CNI), such as tacrolimus (TRL) or cyclosporine (CsA), to reduce CNI-associated nephrotoxicity. ${ }^{2}$

Especially after renal and cardiac transplantation, a significant inverse correlation exists between MPA exposure and the risk of acute rejection. ${ }^{3-8}$ Only few studies on MPA exposure were performed in OLT patients. Similar to renal transplantation, patient and graft survival after OLT are acceptable if MMF is used in combination with CNI, but a change to MMF monotherapy after OLT can be associated with a rate of up to $20 \%$ acute cellular rejection, which-if not treated adequately - can lead to chronic rejection and graft loss. ${ }^{9}$ This may be related to low exposure of MPA., ${ }^{2,10-12}$

In contrast to an initial weight-based dose regimen and therapeutic drug monitoring (TDM) for CNIs, at present most clinics adhere to a fixed dose of MMF, not based on any individual patient's characteristics like age, weight, MPA, or creatinine clearance. ${ }^{13}$ With more experience, it became clear that MMF has a large interpatient variability, with exposures varying 10 -fold, and intrapatient variability over time (especially in the first 6 months posttransplantation). ${ }^{14}$ MPA has complex pharmacokinetics (PKs), and many factors can influence MPA exposure, including kidney and liver function, levels of serum albumin, alterations in absorption, and combination with other immunosuppressive agents. ${ }^{15}$ Recently, studies have been performed to explore feasibility and clinical relevance of MPA trough level monitoring during MMF therapy in solid organ transplantation. ${ }^{16,17}$ Also several limited sampling strategies have been proposed and studied, mostly in renal transplant 
patients, with often 3-5 sampling time points taken in the first 2-6 hours after dosing. ${ }^{18-20}$ Although not specifically investigated, limited sampling strategies with flexible blood sampling using population pharmacokinetic (POP-PK) models and Bayesian estimations may be preferred over strategies with fixed time points based on multivariate analysis, with limited sampling formulas. Le Guellec et $\mathrm{a}^{21}$ developed a limited sampling strategy based on Bayesian estimations as a tool for MPA TDM in renal transplant patients. However, there is limited information on TDM of MPA in liver transplant patients. ${ }^{7,8}$ This probably becomes even more relevant in CNI-free regimens or after CNI dose reduction.

Therefore, the aim of this study was to describe PK of MPA in liver transplant patients. In addition, we were aiming at describing interpatient variability of MPA clearance to further explore the need for TDM in these patients. We studied factors (covariates) like albumin concentration and creatinine clearance that could have an effect on MPA PKs. Finally, a Bayesian TDM strategy for use in patients after liver transplantation was developed using flexible limited sampling models (LSMs) for MPA.

\section{MATERIALS AND METHODS}

Fifty-seven stable patients using MMF who were at least 3 months after OLT were included (median: 231 weeks; range: 49-633). Demographics are listed in Table 1 and Supplemental Digital Content 1 (see Table, http://links.lww.com/TDM/A60). Apart from MMF, 24 patients received tacrolimus ( \pm prednisone) as comedication, 20 received cyclosporine ( \pm prednisone), 2 received sirolimus ( \pm prednisone), whereas 11 patients received only low-dosed glucocorticoids next to $\mathrm{MMF}$

MMF (CellCept, Roche, Basel, Switzerland) was taken twice daily (bid). In our clinic, MMF dosing for liver transplant patients used to be based on fixed dose regimens. Patients started with $500 \mathrm{mg}$ bid and if allowed by absence of leucoand thrombocytopenia and gastrointestinal side effects, the dose was increased to and kept at $1000 \mathrm{mg}$ bid. At the time of this study, 22 patients received $500 \mathrm{mg}$ bid and 28 patients $1000 \mathrm{mg}$ bid. In 7 cases, a deviant dose of $250 \mathrm{mg}$ bid (3 patients), $750 \mathrm{mg}$ bid (3 patients), or $1500 \mathrm{mg}$ bid (1 patient) was given.

After informed consent, all patients visited our clinic for 1 day. Five minutes before administration of the morning dose of MMF, blood samples were obtained for routine chemistry (including liver enzymes, serum creatinine, and serum albumin concentration) and MPA trough ( $\mathrm{C} 0)$ serum concentration.

TABLE 1. Patient Characteristics of Included 57 Patients

\begin{tabular}{lrrrc}
\hline Parameter & Mean & SD & Median & Range \\
\hline Age, yrs & 51 & 12 & 53 & $18-69$ \\
Dose bid, mg & 763 & 281 & 1000 & $250-1500$ \\
Weight, kg & 79 & 18 & 77 & $50-123$ \\
Albumine, g/L & 44 & 4 & 44 & $31-50$ \\
CRCL, mL/min & 78 & 34 & 75 & $16-171$ \\
Time after OLT, wk & 247 & 156 & 231 & $49-633$ \\
\hline
\end{tabular}

Creatinine clearance (CRCL) was calculated with Cockcroft and Gault formula. Patients were instructed to take their evening dose the night before their visit at 10:00 PM. Further blood samples for MPA concentration were collected at $0.5,1,2,3$, and 4 hours in 31 patients and also 6 hours after administration of the morning dose of MMF. In 3 patients, the sample at 0.5 hours was missed. From 10 patients, 2 MPA curves were measured on different days, so in total, 67 curves were available. The missing $\mathrm{C} 12 \mathrm{~h}$ was obtained by extrapolation from $\mathrm{t}=0$ hour to $t=12$ hours, assuming steady-state conditions. ${ }^{22-24}$

Blood was drawn using an indwelling catheter and collected in a vacutainer containing ethylenediaminetetraacetic acid. Plasma MPA concentrations were determined using high performance liquid chromatography. ${ }^{25}$ Imprecision of this method in our laboratory was $4.6 \%-10.1 \%$, depending on MPA concentration. Lower limit of quantitation was $0.2 \mathrm{mg} / \mathrm{L}$. To lower possible influence from meals, patients were instructed to take only a light breakfast - tea and a biscuit - on the morning of measuring the area under the curve (AUC), and no additional food or drinks were allowed until the 2 hours sample (C2).

POP-PK LSMs were developed using the KINPOP module of MW $\backslash$ Pharm, version 3.60 (Mediware, Groningen, The Netherlands; www.mwpharm.nl). ${ }^{26}$ The KINPOP module of MW/Pharm uses an iterative 2-stage Bayesian population procedure to estimate from rich data sets of blood concentration time curves the mathematical description of compartmental PK models. An a priori model acts as a starting point to calculate values for each patient from the available patientspecific data and the a priori population model, leading toward an individualized PK model, indicated as an a posteriori model. The population model is the PK model based on many measurements in many patients. A trapezoidal $\mathrm{AUC}_{0-12 \mathrm{~h}}$ of all 67 curves was calculated with the trapezoidal rule using the software package MW\Pharm.

Individualized PK parameters (individualized PK model based on Bayesian fitting, ie, post hoc values) were obtained. AUCs $(\mathrm{mg} \cdot \mathrm{h} / \mathrm{L})$ based on MPA clearance on single blood sampling time points and combinations of time points were calculated based on the formula: AUC $=\frac{(F \times \text { dose })}{\text { clearance }}$, in which $F$ is bioavailability which was fixed to 1 for MMF since no intravenous data were available. ${ }^{27}$ The dose $(\mathrm{mg})$ is the morning dose of MMF and clearance $(\mathrm{L} / \mathrm{h})$ is apparent clearance (CL/F) of MPA in the absence of information on bioavailability. The $\mathrm{CL} / \mathrm{F}$ was estimated for all patients with Bayesian estimation at different time points and combinations of time points (LSMs).

Statistical analysis on patient data was performed using SPSS 19.0 for Windows (SPSS Inc, Chicago, IL). Results are expressed as mean $\pm \mathrm{SD}$ and as median and range.

Calculated AUCs (with the formula AUC = dose/ clearance), dose, albumin concentration, and CRCL were compared with the trapezoidal $\mathrm{AUC}_{0-12 \mathrm{~h}}$ with the Pearson correlation coefficient. $P$ values $<0.05$ were considered statistically significant.

The ability to describe the trapezoidal $\mathrm{AUC}_{0-12 \mathrm{~h}}$ of the different methods was also investigated by calculating the prediction precision and bias according to Sheiner and Beal. ${ }^{28}$ Prediction bias was calculated as the mean prediction error (MPE); that is, the mean of differences between all $\mathrm{AUCs}_{0-12 \mathrm{~h}}$ 
calculated with the formula (AUCcalc) shown above and all the trapezoidal $\mathrm{AUCs}_{0-12 \mathrm{~h}}$ (AUCtrap): $\left(\frac{(\text { AUCcalc-AUCt rap })}{\text { AUCtrap }}\right) \times 100 \%$. Prediction precision was calculated as the mean absolute prediction error (MAPE); that is, the mean of the absolute differences between all calculated $\mathrm{AUCs}_{0-12 \mathrm{~h}}$ and all the trapezoidal $\mathrm{AUCs}_{0-12 \mathrm{~h}}$ : $\left(\frac{(\text { AUCcalc-AUCtrap })}{\text { AUCtrap }}\right) \times 100 \%$.

MPE and MAPE below 20\% are usually considered acceptable because the lowest possible dose adjustment of MMF is $250 \mathrm{mg}$. Smaller values for MPE and MAPE indicate less bias and greater precision, respectively.

\section{RESULTS}

\section{PK Analysis}

There was a linear relationship between MMF dose and trapezoidal MPA AUC $\left(\mathrm{r}^{2}=0.16, P<0.05\right.$; Fig. 1). Supplemental Digital Content 2 (see Figure, http://links.lww.com/TDM/A59) shows the individual MPA concentration time curves for all patients. There was a wide (8-fold) range in MPA clearances at the same dose level (apparent clearance $=\mathrm{CL} / \mathrm{F}=$ dose/AUCtrap) in the population (500 $\mathrm{mg}$ bid, 8.1-62.5 L/h, and $1000 \mathrm{mg}$ bid, 9.0-71.4 L/h). Looking at possible sources of this variability in MPA clearance, Figure 2 shows the significant inverse relationship between serum albumin concentration and MPA clearance $\left(\mathrm{r}^{2}=0.12, P<0.05\right)$. Specifically, low albumin levels are related to higher MPA clearance. There was also a significant relationship between creatinine clearance and MPA clearance, which is shown in Figure $3\left(\mathrm{r}^{2}=0.24, P<0.05\right)$. The relationship of MPA trough level with the trapezoidal $\mathrm{AUC}_{0-12 \mathrm{~h}}$ is shown in Figure 4.

\section{Comedication}

To explore potential differences in (dose adjusted) MPA AUC between patients with different comedication next to MMF, all patients were divided into 3 groups (cyclosporine, tacrolimus, and no CNIs). These nonsignificant differences are shown in Figure 5 (analysis of variance, $P=0.247$ ). A similar plot could be derived from difference in apparent clearance

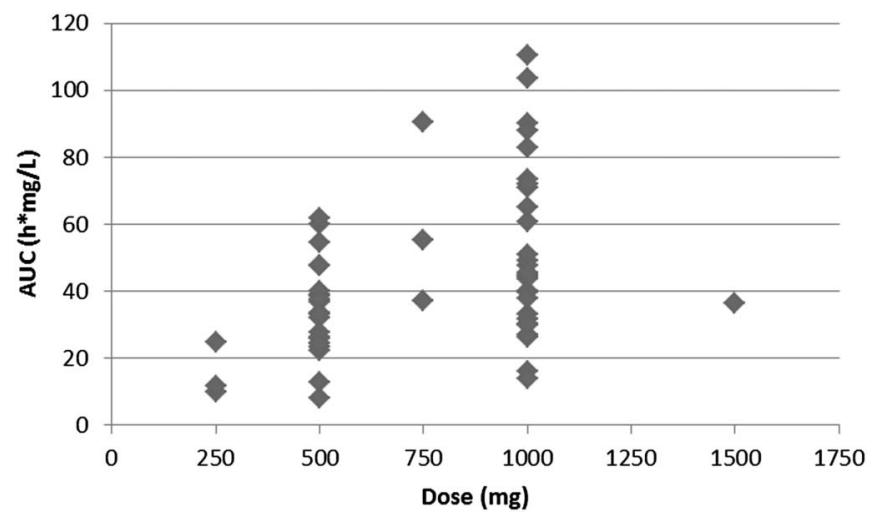

FIGURE 1. MMF dose versus trapezoidal MPA AUC relation$\operatorname{ship}\left(r^{2}=0.16, P<0.05\right)$.

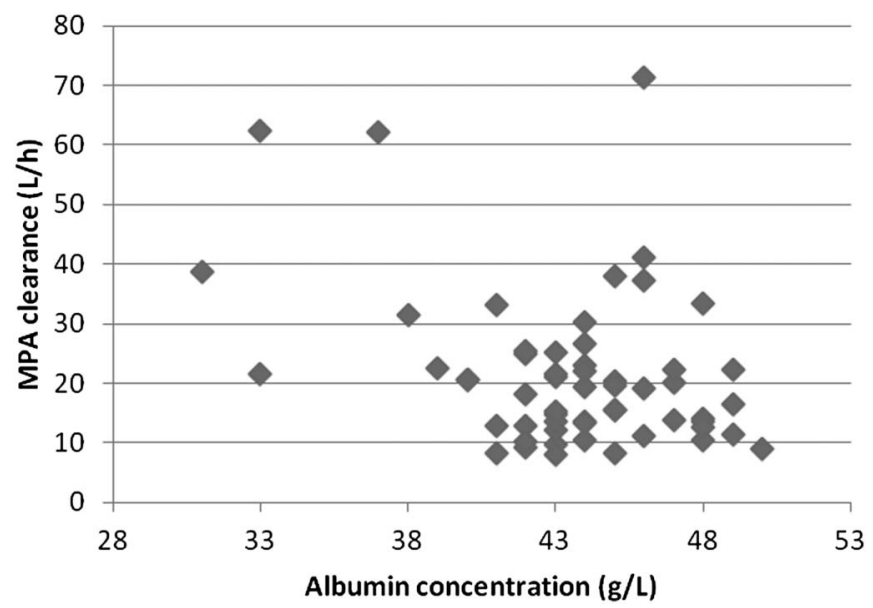

FIGURE 2. Relationship albumin concentration ( $\mathrm{g} / \mathrm{L}$ ) - MPA clearance $(\mathrm{L} / \mathrm{h})\left(\mathrm{r}^{2}=0.12, \mathrm{P}<0.05\right)$.

(data not shown). Figure 6 shows the similar relationship of MPA trough level with dose-adjusted trapezoidal $\mathrm{AUC}_{0-12 \mathrm{~h}}$ for different groups of comedication next to MMF: CsA, TRL, without CNI (analysis of variance, $P=0.112$ ).

\section{Development of LSMs}

An oral 2-compartment model with first-order absorption and lag time described the data adequately. Population parameters for the group of 57 patients were calculated. The best parameters were selected, based on the log-likelihood value of the MW\Pharm, the correlation with trapezoidal MPA AUC and precision and bias. The POP-PK parameters for MMF LSMs are shown in Table 2.

Based on the individualized PK parameters, AUCs for the LSMs based on one- and multiple-point sampling were calculated. Correlations of these calculated AUCs with the trapezoidal $\mathrm{AUC}_{0-12 \mathrm{~h}}$ including bias and precision are shown in Table 3.

Models with single or 2 sampling points show moderate correlation with the trapezoidal AUC, mostly in combination with inacceptable MAPEs. Different combinations of blood sampling time points with 3 or more sampling points show

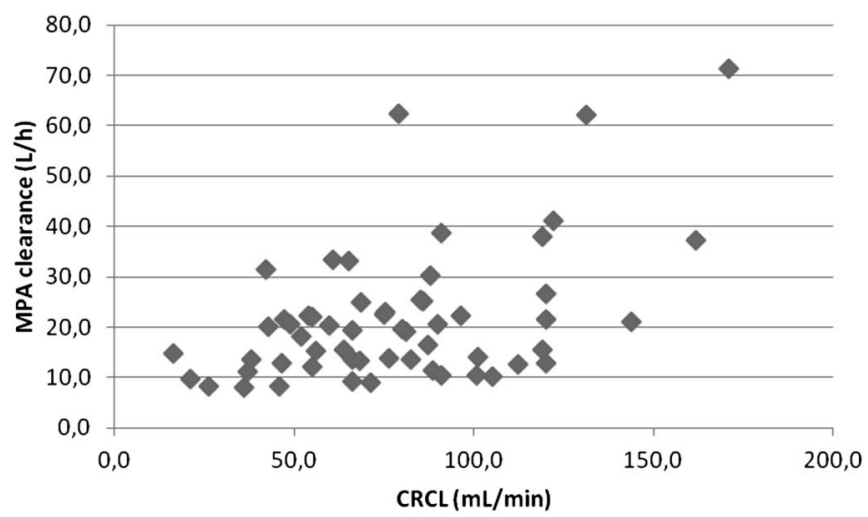

FIGURE 3. Relationship creatinine clearance $(\mathrm{mL} / \mathrm{min})$ - MPA clearance $(L / h)\left(r^{2}=0.24, P<0.05\right)$. 


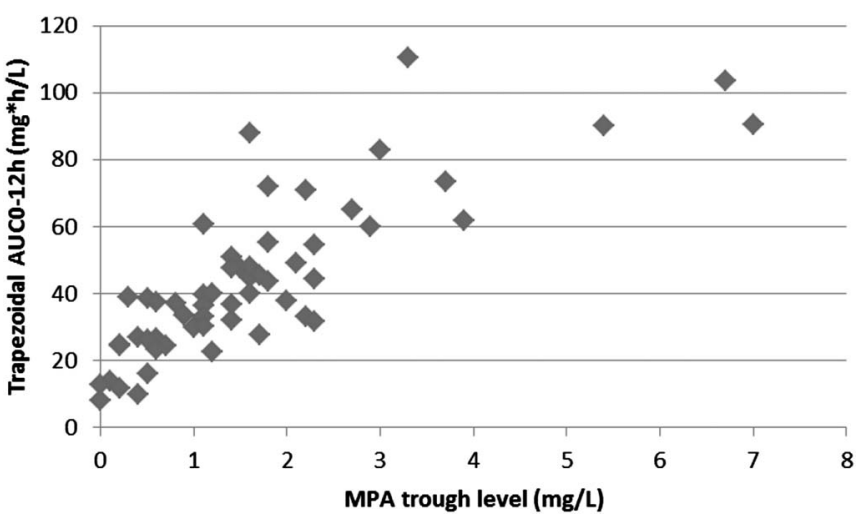

FIGURE 4. Relationship of MPA trough level with the trapezoidal $\mathrm{AUC}_{0-12 \mathrm{~h}}\left(\mathrm{r}^{2}=0.66, \mathrm{P}<0.05\right)$.

good to very good correlations with the trapezoidal AUC. Especially, the combination $0-1 / 2-1-2-3$ hours shows a very good correlation with the trapezoidal $\mathrm{AUC}_{0-12 \mathrm{~h}}$ with acceptable bias and precision $\left(r^{2}=0.92, \mathrm{MPE}=1, \mathrm{MAPE}=13, P<0.05\right)$.

Because 10 extra curves were available, which were not used for model building, we tested the predictability of the trapezoidal AUC using the PK parameters from Table 2. Although this is not a validation, because the same patients of these extra curves were indeed included in model building, very good correlations and MPE/MAPE were observed with the trapezoidal AUC (Table 4).

Correlation of MPA trough levels with the trapezoidal $\mathrm{AUC}_{0-12 \mathrm{~h}}$ for all patients without using any LSMs were moderate, $\mathrm{r}^{2}=0.66(P<0.05)$, Figure 6 . The correlation of trough level $(\mathrm{C} 0)$ with the trapezoidal $\mathrm{AUC}_{0-12 \mathrm{~h}}$, with the use of LSMs, also was moderate $\left(\mathrm{r}^{2}=0.55, P<0.05\right)$, with a 3 -fold trapezoidal AUC difference at random $\mathrm{C} 0$-levels between 1 and $2 \mathrm{mg} / \mathrm{L}$.

Comparing the results of the group including all patients with subgroups of patients with CsA, TRL, CNI, or no CNI did not show a clear advantage over the model with all patients in terms of correlation with the trapezoidal $\mathrm{AUC}_{0-12 \mathrm{~h}}$ and MPE/ MAPE (Table 3).

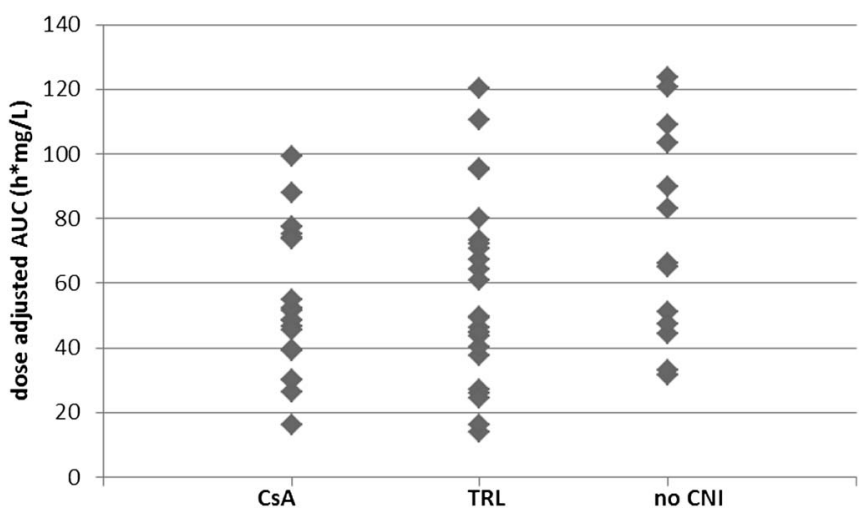

FIGURE 5. Patients with cyclosporine, patients with tacrolimus, and patients without CNIs as comedication next to MMF and their nonsignificant difference in dose-adjusted AUC $(P=0.247)$.
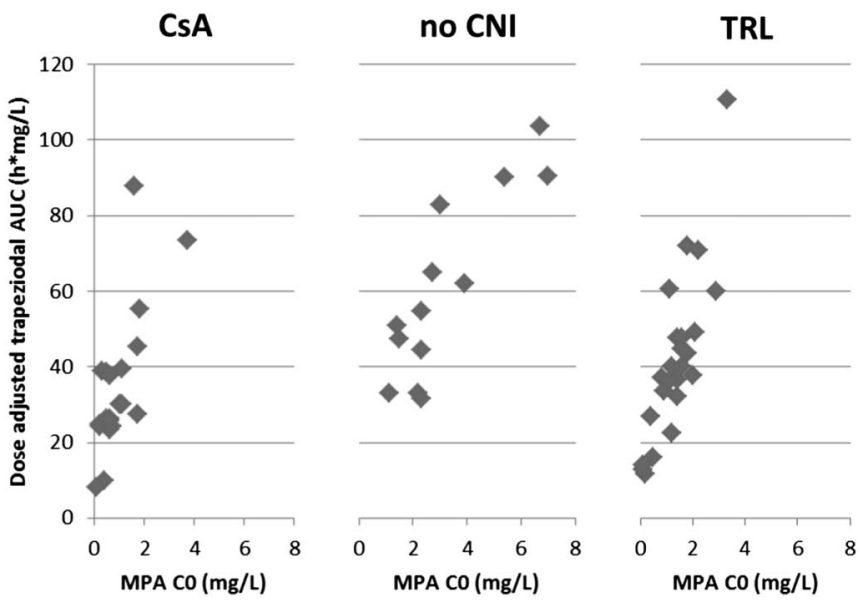

FIGURE 6. Relationship of MPA trough level with doseadjusted trapezoidal $\mathrm{AUC}_{0-12 \mathrm{~h}}$ for different groups of comedication next to MMF: CsA (Pearson $\mathrm{R}=0.441, P=0.05$ ), TRL (Pearson $\mathrm{R}=0.799, P<0.01$ ), or without $\mathrm{CNI}$ (no $\mathrm{CNI}$ ) (Pearson $\mathrm{R}=0.711, P<0.01$ ) (analysis of variance, $P=0.121$ ).

\section{DISCUSSION}

We describe the PKs of MPA in liver transplant patients. Noncompartmental PK analysis confirmed an earlier described linear relationship between MMF dose and the area under the concentration time curve (AUC). However, an 8 -fold variability in dose-corrected systemic exposure and therefore apparent oral clearance was also observed, illustrating a high interpatient variability in the PK of MPA. To some extent, this variability is explained by earlier described factors affecting MPA PK such as serum albumin and CRCL. In the current data, there is an inverse correlation between serum albumin and MPA clearance. Due to the absence of collection of unbound MPA concentration data and the specific selection of patients from this study, no definite conclusions can be drawn regarding the relationship between serum albumin concentration and MPA exposure. However, the relationship we describe is in line with previous observations: recently, lower serum albumin was related to higher free MPA and lower total MPA in renal transplantation. ${ }^{29}$ van Hest et $\mathrm{al}^{30}$ also found that the exposure to total MPA, as determined by MPA clearance, significantly increased with increasing renal function, albumin level and hemoglobin, and decreasing cyclosporin predose level. No significant difference of doseadjusted MPA AUC of the 3 groups of comedication existed (cyclosporine, tacrolimus, no CNIs).

TABLE 2. Population PK Parameters for MMF LSMs

\begin{tabular}{lcr}
\hline Parameters & Population & \multicolumn{1}{c}{ \pm} \\
\hline Apparent clearance, $\mathrm{L} / \mathrm{h}$ per $70 \mathrm{~kg}$ & 17.43 & 8.78 \\
Volume (central), L/kg & 0.27 & 0.42 \\
Intercompartimental clearance, $\mathrm{L} / \mathrm{h}$ per $70 \mathrm{~kg}$ & 21.70 & 21.81 \\
Volume (peripheral), L/kg & 7.56 & 17.40 \\
Absorption rate constant, $/ \mathrm{h}$ & 6.28 & 10.96 \\
Oral bioavailability & 1 & Fixed \\
Lag time, h & 0.32 & 0.28 \\
\hline
\end{tabular}


TABLE 3. Correlations of MPA AUC Calculated With Gold Standard (Trapezoidal) AUC $\mathrm{C}_{0-12 \mathrm{~h}}$ for Models Based on One- and Multiple-Point Sampling $(P<0.05)$

\begin{tabular}{|c|c|c|c|c|c|c|c|c|c|c|c|c|c|c|c|}
\hline LSM & \multicolumn{3}{|c|}{$\begin{array}{l}\text { Total Group } \\
(\mathbf{n}=\mathbf{5 7})\end{array}$} & \multicolumn{3}{|c|}{$\begin{array}{c}\text { CsA } \\
(n=20)\end{array}$} & \multicolumn{3}{|c|}{$\begin{array}{c}\text { TRL } \\
(n=24)\end{array}$} & \multicolumn{3}{|c|}{$\begin{array}{c}\text { CNI } \\
(n=44)\end{array}$} & \multicolumn{3}{|c|}{$\begin{array}{l}\text { No CNI } \\
(n=13)\end{array}$} \\
\hline $\mathrm{cl0}$ & 0.56 & 9 & 33 & 0.32 & -1 & 33 & 0.36 & 18 & 43 & 0.37 & 11 & 38 & 0.79 & 4 & 18 \\
\hline cl1 & 0.26 & 6 & 38 & 0.17 & 4 & 34 & 0.21 & 20 & 49 & 0.24 & 14 & 41 & 0.27 & -18 & 29 \\
\hline $\mathrm{cl} 2$ & 0.33 & 21 & 44 & 0.22 & 17 & 40 & 0.26 & 37 & 58 & 0.30 & 29 & 49 & 0.32 & -7 & 26 \\
\hline $\mathrm{cl} 3$ & 0.33 & 35 & 49 & 0.09 & 46 & 62 & 0.37 & 39 & 53 & 0.25 & 43 & 56 & 0.64 & 6 & 22 \\
\hline cl02 & 0.70 & 7 & 27 & 0.50 & -1 & 27 & 0.60 & 14 & 33 & 0.56 & 8 & 30 & 0.87 & 6 & 15 \\
\hline cl03 & 0.74 & 12 & 29 & 0.75 & 9 & 35 & 0.64 & 13 & 30 & 0.66 & 12 & 32 & 0.78 & 14 & 21 \\
\hline cl12 & 0.25 & 15 & 40 & 0.22 & 21 & 40 & 0.31 & 27 & 48 & 0.27 & 24 & 44 & 0.40 & -15 & 24 \\
\hline cl13 & 0.35 & 37 & 49 & 0.54 & 50 & 57 & 0.41 & 39 & 52 & 0.41 & 44 & 54 & 0.35 & 15 & 33 \\
\hline $\mathrm{cl} 23$ & 0.56 & 39 & 48 & 0.64 & 45 & 54 & 0.36 & 44 & 56 & 0.48 & 45 & 54 & 0.69 & 20 & 27 \\
\hline cl012 & 0.65 & 6 & 29 & 0.41 & 2 & 32 & 0.57 & 6 & 31 & 0.51 & 5 & 31 & 0.79 & 10 & 20 \\
\hline $\operatorname{cl} 0^{1 / 23}$ & 0.73 & 12 & 25 & 0.40 & 19 & 38 & 0.81 & 4 & 20 & 0.57 & 12 & 27 & 0.83 & 15 & 18 \\
\hline $\operatorname{cl} 0^{1 / 2} 12$ & 0.76 & -1 & 22 & 0.80 & -7 & 17 & 0.52 & 0 & 29 & 0.67 & -4 & 23 & 0.79 & 7 & 17 \\
\hline $\mathrm{cl} 0^{1 / 223}$ & 0.81 & 11 & 23 & 0.74 & 8 & 25 & 0.60 & 12 & 29 & 0.69 & 11 & 26 & 0.92 & 12 & 14 \\
\hline cl0123 & 0.81 & 3 & 18 & 0.79 & 7 & 26 & 0.70 & 3 & 18 & 0.75 & 3 & 21 & 0.88 & 4 & 12 \\
\hline $\mathrm{cl} 0^{1 / 2} 123$ & 0.92 & 1 & 13 & 0.94 & 2 & 15 & 0.86 & -3 & 14 & 0.89 & -1 & 14 & 0.92 & 4 & 8 \\
\hline $\mathrm{cl} 0^{1 / 2} 1234$ & 0.94 & -1 & 11 & 0.92 & -4 & 12 & 0.94 & -3 & 11 & 0.93 & -3 & 11 & 0.95 & 7 & 10 \\
\hline
\end{tabular}

CsA, group with CsA as comedication next to MMF; CNI, group with a CNI as comedication next to MMF; no CNI, group with no CNI as comedication next to MMF; TRL, group with TRL as comedication next to MMF.

This analysis was the basis for a proposal to improve TDM for MMF after liver transplantation: we developed LSMs for MPA TDM in stable liver transplant patients. The program used for Bayesian estimations is a 2-stage approach, which is able to predict PK parameters adequately in strictly defined populations.

TABLE 4. Correlations of MPA AUC Calculated With Gold Standard (Trapezoidal) $A_{U C} C_{0-12 h}$ for 10 Available Extra Curves

\begin{tabular}{|c|c|c|c|}
\hline LSM & $\mathbf{r}^{2}$ & MPE & MAPE \\
\hline cl0 & 0.59 & 29 & 37 \\
\hline cl01 & 0.62 & 17 & 33 \\
\hline cl02 & 0.72 & 27 & 35 \\
\hline cl03 & 0.67 & 34 & 40 \\
\hline cl012 & 0.77 & 12 & 25 \\
\hline cl013 & 0.67 & 28 & 39 \\
\hline cl023 & 0.71 & 35 & 40 \\
\hline $\mathrm{cl} 01 / 21$ & 0.54 & 8 & 35 \\
\hline $\mathrm{cl} 01 / 22$ & 0.82 & 15 & 26 \\
\hline $\mathrm{cl} 01 / 23$ & 0.86 & 20 & 27 \\
\hline $\mathrm{cl} 1 \frac{1}{2} 12$ & 0.83 & -2 & 18 \\
\hline $\mathrm{cl} 01 / 223$ & 0.87 & 20 & 26 \\
\hline cl0123 & 0.78 & 23 & 32 \\
\hline $\mathrm{cl} 01 / 2123$ & 0.97 & 12 & 16 \\
\hline cl01/21234 & 0.85 & 8 & 21 \\
\hline
\end{tabular}

Some combinations of time points showed excellent correlation with the trapezoidal $\mathrm{AUC}_{0-12 \mathrm{~h}}$ with low prediction error when using a LSM. Because these Bayesian models have no need for fixed time points, they are very flexible and easy to use in daily practice in the outpatient clinic, as we have shown before for cyclosporine and tacrolimus monitoring. ${ }^{31,32}$ The trough level with and without the model demonstrated a moderate correlation with the trapezoidal AUC.

The imprecision with a trough level approach is shown in Figures 4 and 6. This large difference in AUC at a measured trough level (ie, 0.5 or $1.75 \mathrm{mg} / \mathrm{L}$ ) is a reflection of the large interpatient variability and this is a serious problem with a trough level approach.

There are several reasons for introducing TDM of MMF in daily practice. MPA levels are related to efficacy (rejection) and safety (adverse events). ${ }^{3-7,33}$ Yau et $\mathrm{al}^{34}$ already concluded that fixed dose regimens of MMF may not be optimal for all patients. Others also demonstrated a significant interpatient variation in MPA PKs due to factors such as renal function, albumin level, and (cyclosporine) comedication. $^{30,35-38}$ One-third of the patients on cyclosporine receiving fixed dose MMF immediately after renal transplantation were underdosed when the AUC was calculated, and this was related to a higher incidence of rejection. ${ }^{39}$ Furthermore, an increase of Cmax and AUC of MPA in renal transplant recipients in the months after transplantation is described. ${ }^{27}$ This may require dose adjustments. 
Calcineurin inhibitors are widely used after organ transplantation. A disadvantage of these drugs is their nephrotoxicity. MMF, in contrast to CNIs, does not cause renal damage. Its use may allow the lowering or even discontinuation of CNI dosing ${ }^{40,41}$ which may lead to better kidney function in the long term. ${ }^{11,42}$ However, CNI cessation or dose reduction with fixed dose MMF monotherapy (or with steroids) after liver transplantation may lead to acute or even chronic rejection in a significant percentage of the patients. ${ }^{2,10-12}$ A solid TDMbased dose guiding strategy for MPA may reduce these risks.

A review article from Kaplan ${ }^{17}$ concluded that the contribution of TDM for MPA in the investigated studies remains unproven and that results of large randomized controlled trials were awaited. Another review article from Arns et $\mathrm{al}^{16}$ concluded that there still was no clear support for a substantial clinical benefit of TDM, but that MPA AUC might be more reliable than predose (C0) MPA levels. Zicheng et $\mathrm{al}^{8}$ developed limited sampling algorithms for implementation of MPA monitoring in liver transplantation necessitating exactly timed blood sampling. In the roundtable meeting of van Gelder et $\mathrm{al}^{15}$ different limited sampling strategies, mostly algorithms, for monitoring MPA were described as good estimators of $\mathrm{AUC}_{0-12 \mathrm{~h}}$ with acceptable predictive performance. Le Meur et $\mathrm{al}^{14}$ concluded in the APOMYGRE trial that therapeutic MPA monitoring using a limited sampling strategy can reduce the risk of treatment failure and acute rejection in renal allograft recipients in the 12 months posttransplant, compared with fixed dose MMF, with no increase in adverse events.

The distinction between cyclosporine and no-cyclosporine as comedication of MMF is described in different studies. ${ }^{30,43-46}$ Cyclosporine has an influence on MPA clearance by disrupting the enterohepatic cycle, leading to lower MPA exposure. ${ }^{25,47}$ A limitation of our study is the absence of blood sampling time points between 6 and 12 hours after dosing MMF, exactly the time in which the enterohepatic recirculation may occur. Due to these missing values, we could not entirely take the enterohepatic cycle into account, which may mean that the MPA AUCs in patients using cyclosporine may be slightly higher than calculated in our study. Regarding the concentration before dosing (C0-level), no significant difference between the patients with cyclosporine and tacrolimus was found $(P=0.122)$. On future validation with more patients, covariate analysis of comedication next to MMF can be taken into account. Because of possible disturbances in bile production and flow, the influence of the enterohepatic cycle might be different in liver transplant patients compared with renal transplant recipients. ${ }^{25} \mathrm{We}$ assumed that there were no such disturbances in these stable patients more than 3 months after OLT, as supported by a similar dose-adjusted MPA AUC for the groups with comedication with tacrolimus and cyclosporine.

Another limitation is that the model is based on a limited number of patients $(\mathrm{n}=57)$. However, it performed well on 10 different curves from the same patients, and we are planning to validate this model on a larger independent patient cohort. The role of trough level monitoring in combination with a POP-PK model needs further validation on a larger data set. The model with sampling at $0-1 / 2-1-2-3$ hours for the total group of patients, showed good correlations with trapezoidal $\mathrm{AUC}_{0-12 \mathrm{~h}}$ and acceptable bias and precision. To adequately estimate the $\mathrm{AUC}_{0-12 \mathrm{~h}}$ data beyond a single trough level are needed, with probably at least 4 time points. Prospective validation is needed, measuring samples over a 24-hour period of time.

In practice, monitoring with multiple time points is feasible, as we have shown before with cyclosporine monitoring after $\mathrm{OLT}^{48}{ }^{48}$ It only requires a simple computer program the like of which is available in most hospital pharmacies.

No target ranges for the MPA AUC, especially for liver transplantation patients, have been developed yet. In the scarce literature about TDM of MPA after liver transplantation, Tredger et $\mathrm{al}^{7}$ suggests a therapeutic range of $1-3.5 \mathrm{mg} / \mathrm{L}$ for trough level monitoring to prevent acute rejection and to lower adverse effects, like infection, leukopenia, and gastrointestinal disturbances. For renal transplantation in the early posttransplant period, an $\mathrm{AUC}_{0-12 \mathrm{~h}}$ target of $40 \mathrm{mg} \cdot \mathrm{h} \cdot \mathrm{L}^{-1}$, or a range of $30-60 \mathrm{mg} \cdot \mathrm{h} \cdot \mathrm{L}^{-1}$, is adhered to in the presence of a CNI. ${ }^{15}$ A target AUC of $75 \mathrm{mg} \cdot \mathrm{h} \cdot \mathrm{L}^{-1}$ (range: $60-90 \mathrm{mg} \cdot \mathrm{h} \cdot \mathrm{L}^{-1}$ ) for kidney transplant recipients on MMF allows safe cyclosporine withdrawal in most patients. ${ }^{49}$ For the moment we suggest-in the absence of sufficient data from clinical studies - to use similar targets in liver transplantation as in renal transplantation.

\section{CONCLUSIONS}

In conclusion, with the flexible and accurate Bayesian LSM for MMF (we propose a model with sampling times 0-1/ 2-1-2-3 hours), a tool is provided for improved TDM-based dose guiding of MMF in liver transplant patients. Its use in combination with the suggested target ranges may allow safe lowering or discontinuation of CNIs after OLT to improve renal function while avoiding rejection. Prospective validation and assessment of clinical relevance of our model are planned.

\section{ACKNOWLEDGMENT}

This project was sponsored by Fonds NutsOhra, www. fondsnutsohra.nl.

\section{REFERENCES}

1. Allison AC, Eugui EM. The design and development of an immunosuppressive drug, mycophenolate mofetil. Springer Semin Immunopathol. 1993; 14:353-380.

2. Reich DJ, Clavien PA, Hodge EE. Mycophenolate mofetil for renal dysfunction in liver transplant recipients on cyclosporine or tacrolimus: randomized, prospective, multicenter pilot study results. Transplantation. 2005;80:18-25.

3. Kiberd BA, Lawen J, Fraser AD, et al. Early adequate mycophenolic acid exposure is associated with less rejection in kidney transplantation. $\mathrm{Am} \mathrm{J}$ Transplant. 2004;4:1079-1083.

4. Pillans PI, Rigby RJ, Kubler P, et al. A retrospective analysis of mycophenolic acid and cyclosporin concentrations with acute rejection in renal transplant recipients. Clin Biochem. 2001;34:77-81.

5. DeNofrio D, Loh E, Kao A, et al. Mycophenolic acid concentrations are associated with cardiac allograft rejection. J Heart Lung Transplant. 2000;19:1071-1076.

6. Yamani MH, Starling RC, Goormastic M, et al. The impact of routine mycophenolate mofetil drug monitoring on the treatment of cardiac allograft rejection. Transplantation. 2000;69:2326-2330.

7. Tredger JM, Brown NW, Adams J, et al. Monitoring mycophenolate in liver transplant recipients: toward a therapeutic range. Liver Transpl. 2004;10:492-502.

8. Zicheng $\mathrm{Y}$, Weixia $\mathrm{Z}$, Hao $\mathrm{C}$, et al. Limited sampling strategy for the estimation of mycophenolic acid area under the plasma concentration-time 
curve in adult patients undergoing liver transplant. Ther Drug Monit. 2007; 29:207-214

9. Wiesner R, Rabkin J, Klintmalm G, et al. A randomized double-blind comparative study of mycophenolate mofetil and azathioprine in combination with cyclosporine and corticosteroids in primary liver transplant recipients. Liver Transpl. 2001;7:442-450.

10. Bilbao I, Castells L, Rojas L, et al. Immunosuppression based on mycophenolate mofetil in stable liver transplanted patients. Int Immunopharmacol. 2006;6:1977-1983.

11. Moreno Planas JM, Cuervas-Mons Martinez V, Rubio Gonzalez E, et al. Mycophenolate mofetil can be used as monotherapy late after liver transplantation. Am J Transplant. 2004;4:1650-1655.

12. Fairbanks KD, Thuluvath PJ. Mycophenolate mofetil monotherapy in liver transplantation: a single center experience. Liver Transpl. 2004; 10:1189-1194.

13. van Gelder T, Hilbrands LB, Vanrenterghem Y, et al. A randomized doubleblind, multicenter plasma concentration controlled study of the safety and efficacy of oral mycophenolate mofetil for the prevention of acute rejection after kidney transplantation. Transplantation. 1999;68:261-266.

14. Le Meur Y, Büchler M, Thierry A, et al. Individualized mycophenolate mofetil dosing based on drug exposure significantly improves patient outcomes after renal transplantation. Am J Transplant. 2007;7:2496-2503.

15. van Gelder T, Le Meur Y, Shaw LM, et al. Therapeutic drug monitoring of mycophenolate mofetil in transplantation. Ther Drug Monit. 2006;28: $145-154$.

16. Arns W, Cibrik DM, Walker RG, et al. Therapeutic drug monitoring of mycophenolic acid in solid organ transplant patients treated with mycophenolate mofetil: review of the literature. Transplantation. 2006;82:1004-1012.

17. Kaplan B. Mycophenolic acid trough level monitoring in solid organ transplant recipients treated with mycophenolate mofetil: association with clinical outcome. Curr Med Res Opin. 2006;22:2355-2364.

18. Pawinski T, Hale M, Korecka M, et al. Limited sampling strategy for the estimation of mycophenolic acid area under the curve in adult renal transplant patients treated with concomitant tacrolimus. Clin Chem. 2002;48:1497-1504.

19. Shaw LM, Holt DW, Oellerich M, et al. Current issues in therapeutic drug monitoring of mycophenolic acid: report of a roundtable discussion. Ther Drug Monit. 2001;23:305-315.

20. Filler G. Abbreviated mycophenolic acid AUC from C0, C1, C2, and C4 is preferable in children after renal transplantation on mycophenolate mofetil and tacrolimus therapy. Transpl Int. 2004;17:120-125.

21. Le Guellec C, Bourgoin H, Büchler M, et al. Population pharmacokinetics and Bayesian estimation of mycophenolic acid concentrations in stable renal transplant patients. Clin Pharmacokinet. 2004;43:253-266.

22. Filler G, Mai I. Limited sampling strategy for mycophenolic acid area under the curve. Ther Drug Monit. 2000;22:169-173.

23. Mardigyan V, Tchervenkov J, Metrakos P. Best single time points as surrogates to the tacrolimus and mycophenolic acid area under the curve in adult liver transplant patients beyond 12 months of transplantation. Clin Ther. 2005;27:463-469.

24. Behrend M. Mycophenolate mofetil: suggested guidelines for use in kidney transplantation. BioDrugs. 2001;15:37-53.

25. Cremers S, Schoemaker R, Scholten E, et al. Characterizing the role of enterohepatic recycling in the interactions between mycophenolate mofetil and calcineurin inhibitors in renal transplant patients by pharmacokinetic modelling. Br J Clin Pharmacol. 2005;60:249-256.

26. Proost JH. Adaptive control of drug dosage regimens using maximum a posteriori probability Bayesian fitting. Int $J$ Clin Pharmacol Ther. 1995;33:531-536

27. Bullingham RE, Nicholls AJ, Kamm BR. Clinical pharmacokinetics of mycophenolate mofetil. Clin Pharmacokinet. 1998;34:429-455.

28. Sheiner LB, Beal SL. Some suggestions for measuring predictive performance. J Pharmacokinet Biopharm. 1981;9:503-512.

29. de Winter BCM, van Gelder T, Sombogaard F, et al. Pharmacokinetic role of protein binding of mycophenolic acid and its glucuronide metab- olite in renal transplant recipients. J Pharmacokinet Pharmacodyn. 2009; 36:541-564.

30. van Hest RM, Mathot RAA, Pescovitz MD, et al. Explaining variability in mycophenolic acid exposure to optimize mycophenolate mofetil dosing: a population pharmacokinetic meta-analysis of mycophenolic acid in renal transplant recipients. J Am Soc Nephrol. 2006;17:871-880.

31. Langers P, Cremers SC, den Hartigh J, et al. Easy-to-use, accurate and flexible individualized Bayesian limited sampling method without fixed time points for ciclosporin monitoring after liver transplantation. Aliment Pharmacol Ther. 2005;21:549-557.

32. Langers P, Press RR, den Hartigh J, et al. Flexible limited sampling model for monitoring tacrolimus in stable patients having undergone liver transplantation with samples 4 to 6 hours after dosing is superior to trough concentration. Ther Drug Monit. 2008;30:456-461.

33. Borrows R, Chusney G, James A, et al. Determinants of mycophenolic acid levels after renal transplantation. Ther Drug Monit. 2005;27:442-450.

34. Yau WP, Vathsala A, Lou HX, et al. Is a standard fixed dose of mycophenolate mofetil ideal for all patients? Nephrol Dial Transplant. 2007; 22:3638-3645.

35. Borrows R, Chusney G, Loucaidou M, et al. Mycophenolic acid 12-h trough level monitoring in renal transplantation: association with acute rejection and toxicity. Am J Transplant. 2006;6:121-128.

36. van Hest RM, van Gelder T, Vulto AG, et al. Population pharmacokinetics of mycophenolic acid in renal transplant recipients. Clin Pharmacokinet. 2005;44:1083-1096.

37. Kuriata-Kordek M, Boratynska M, Falkiewicz K, et al. The influence of calcineurin inhibitors on mycophenolic acid pharmacokinetics. Transplant Proc. 2003;35:2369-2371.

38. Cattaneo D, Gaspari F, Ferrari S, et al. Pharmacokinetics help optimizing mycophenolate mofetil dosing in kidney transplant patients. Clin Transplant. 2001;15:402-409.

39. Gaston RS, Kaplan B, Shah T, et al. Fixed- or controlled-dose mycophenolate mofetil with standard- or reduced-dose calcineurin inhibitors: the Opticept trial. Am J Transplant. 2009;9:1607-1619.

40. Flechner SM. Minimizing calcineurin inhibitor drugs in renal transplantation. Transplant Proc. 2003;35:118S-121S.

41. Créput C, Blandin F, Deroure B, et al. Long-term effects of calcineurin inhibitor conversion to mycophenolate mofetil on renal function after liver transplantation. Liver Transpl. 2007;13:1004-1010.

42. Pfitzmann R, Klupp J, Langrehr JM, et al. Mycophenolatemofetil for immunosuppression after liver transplantation: a follow-up study of 191 patients. Transplantation. 2003;76:130-136.

43. van Gelder T, Klupp J, Barten MJ, et al. Comparison of the effects of tacrolimus and cyclosporine on the pharmacokinetics of mycophenolic acid. Ther Drug Monit. 2001;23:119-128.

44. Smak Gregoor PJ, van Gelder T, Hesse CJ, et al. Mycophenolic acid plasma concentrations in kidney allograft recipients with or without cyclosporin: a cross-sectional study. Nephrol Dial Transplant. 1999; 14:706-708.

45. Filler G, Zimmering M, Mai I. Pharmacokinetics of mycophenolate mofetil are influenced by concomitant immunosuppression. Pediatr Nephrol. 2000; 14:100-104.

46. Patel CG, Harmon M, Gohh RY, et al. Concentrations of mycophenolic acid and glucuronide metabolites under concomitant therapy with cyclosporine or tacrolimus. Ther Drug Monit. 2007;29:87-95.

47. Hesselink DA, van Hest RM, Mathot RA, et al. Cyclosporine interacts with mycophenolic acid by inhibiting the multidrug resistance-associated protein 2. Am J Transplant. 2005;5:987-994.

48. Langers $\mathrm{P}$, Cremers SC, den Hartigh J, et al. Individualized population pharmacokinetic model with limited sampling for cyclosporine monitoring after liver transplantation in clinical practice. Aliment Pharmacol Ther. 2007;26:1447-1454.

49. Mourer JS, den Hartigh J, van Zwet EW, et al. Randomized trial comparing late concentration-controlled calcineurin inhibitor or mycophenolate mofetil withdrawal. Transplantation. 2012;93:887-894. 